\title{
Predicting Intended Movement Direction Using EEG from Human Posterior Parietal Cortex
}

\author{
Yijun Wang and Scott Makeig \\ Swartz Center for Computational Neuroscience, Institute for Neural Computation, \\ University of California, San Diego, USA \\ \{yijun, scott $\} @$ sccn.ucsd.edu
}

\begin{abstract}
The posterior parietal cortex (PPC) plays an important role in motor planning and execution. Here, we investigated whether noninvasive electroencephalographic (EEG) signals recorded from the human PPC can be used to decode intended movement direction. To this end, we recorded wholehead EEG with a delayed saccade-or-reach task and found direction-related modulation of event-related potentials (ERPs) in the PPC. Using parietal EEG components extracted by independent component analysis (ICA), we obtained an average accuracy of $80.25 \%$ on four subjects in binary single-trial EEG classification (left versus right). These results show that in the PPC, neuronal activity associated with different movement directions can be distinguished using EEG recording and might, thus, be used to drive a noninvasive brainmachine interface (BMI).
\end{abstract}

Key words: posterior parietal cortex (PPC); electroencephalography (EEG); independent component analysis (ICA); brain-machine interface (BMI).

\section{Introduction}

In current brain-machine interface (BMI) research, predicting intended movement trajectory is a widely proposed method for controlling prosthetic limbs [1]. Most tested systems for monkey and human subjects are based on neuronal activities recorded in the primary motor cortex (M1), where neuron firing patterns encode direction information about limb movement [2-4]. In neuroscience, it is also well known that the parietal cortex plays an important role in movement planning, being involved in sensorimotor transformations from visual input to motor execution. For instance, the posterior parietal cortex (PPC) is critically involved in visuo-motor control of visually guided reaching movements, continuously updating reaching movements to the visual target. According to its role in motor planning, the parietal cortex may provide another way to decode intended movement direction, which can be potential for BMI applications. In recent monkey studies, direction decoding of eye and hand movements has been realized using neuronal signals in the PPC [5]. The PPC of monkey brain can be further divided into subareas for different action 


\section{Yijun Wang and Scott Makeig}

planning, e.g., the lateral intraparietal area (LIP) for saccades and the parietal reach region (PRR) for reaches.

For real-world application, non-invasive brain-computer interfaces (BCIs) based on electroencephalographic (EEG) signals are more practical than invasive BMIs, whose human applications are seriously limited by questions about the safety and durability of implanted electrodes [6-8]. Various EEG signals have been employed to build different kinds of EEG-based BCI systems, e.g., P300 evoked potential, visual evoked potential (VEP), and mu/beta rhythm power [7]. So far, movement direction decoding using noninvasive methods has been tried only in very few studies [9] [10]. In [9], a machine learning paradigm was successfully applied to discriminate movement directions using single-trial EEG data recorded during natural and delayed reaching tasks. However, the functional brain components contributing most to classification have not been specified in this study, and therefore the underlying brain dynamics related to direction coding are still unclear. Recently, a magnetoencephalography (MEG) study showed that the direction of hand movements can be inferred from brain activities [10]. In their study, movement directions were decoded based on power modulation in the low-frequency band $(<7 \mathrm{~Hz})$ using MEG activities from the motor area. To the best of our knowledge, intended direction decoding in the PPC based on EEG recordings has been rarely studied, and is ignored in current BCI research. In the present study, we investigated brain activity in the human PPC during directional movement planning using multichannel event-related potentials (ERPs), and propose a BCI scheme based on single-trial EEG classification.

\section{Method}

\subsection{Subjects}

Four healthy, right-handed participants ( 3 males and 1 female, mean age 25 years) with normal or corrected-to-normal vision performed this experiment. All participants were asked to read and sign an informed consent form approved by the UCSD Human Research Protections Program before participating in the study.

\subsection{Stimuli and Procedure}

During execution of eye or hand movements, movement artifacts including electrooculographic (EOG) and electromyographic (EMG) signals also include direction information about the attended movement. To obtain clean brain signals not including such information, therefore, a delayed saccade-or-reach task was used in this study, allowing us to look for direction information in the EEG during the phase of movement planning. The experiment was comprised of nine conditions differing by movement type (saccade to target, reach without eye movement, or visually guided 
reach) and movement direction (left, center, or right). Each task was indicated to the subject by, first, giving an effector cue telling the type of action to be performed, followed by a direction cue and, finally, by an imperative action cue. Subjects were seated comfortably in an armchair at a distance of $40 \mathrm{~cm}$ from a 19 -inch touch screen. A chin rest was used to help them maintain head position.

Subjects used their right hand to perform reach tasks. At the beginning of each trial, the subject's forearm rested on the table with index finger holding down a key on a keypad placed $30 \mathrm{~cm}$ in front of screen center. The sequence of visual cues in each trial is shown in Fig.1(a). At the beginning of a trial, a fixation cross $\left(0.65^{\circ} \times 0.65^{\circ}\right)$ was displayed in the center of the screen plus three red crosses $\left(0.65^{\circ} \times 0.65^{\circ}\right)$ indicating potential target positions. The left and right targets had a vertical distance of $6^{\circ}$ and a horizontal distance of $15^{\circ}$ from the central fixation cross; the central target was $12^{\circ}$ upwards. After $500 \mathrm{~ms}$, an effector cue $\left(0.5^{\circ} \times 0.5^{\circ}\right.$, rectangle, ellipse indicating hand and eye movements respectively, see Fig.1(b)) appeared at screen center for $1000 \mathrm{~ms}$. Next, a central direction cue $\left(0.65^{\circ} \times 0.65^{\circ},-\right.$, $\perp$, $\vdash$ for left, center, and right respectively) was presented for $700 \mathrm{~ms}$. Subjects were asked to maintain fixation on the central cue until they started their response, to perform the indicated response as quickly as possible following the disappearance of the direction cue (and reappearance of the fixation cross), and finally to return to their initial (key-down) position. Total trial duration amounted to $3500 \sim 4000 \mathrm{~ms}$.

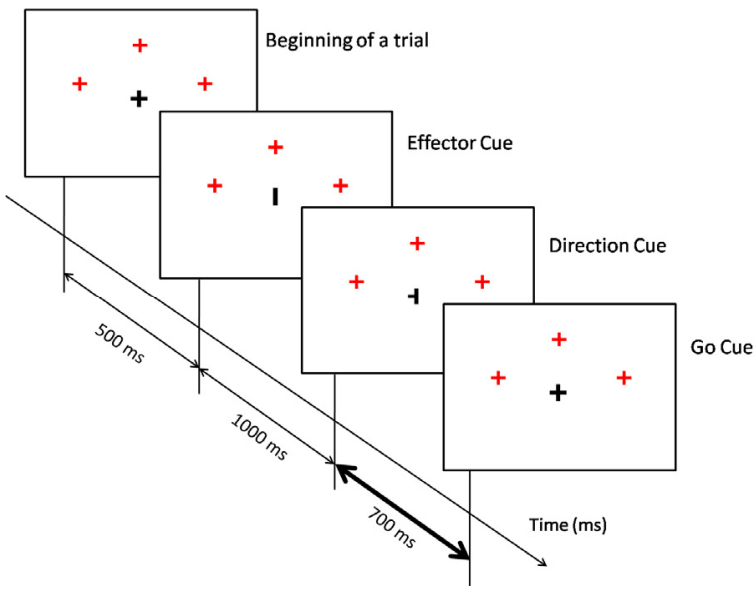

(a)

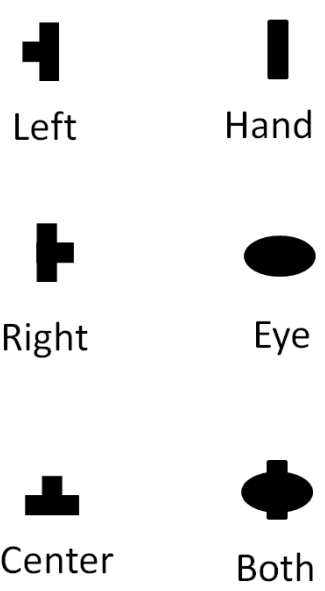

(b)

Fig. 1. (a) Time sequence of cue presentation in a trial and (b) visual cues used to indicate effector and direction of a task. In each trial, three central cues (first, effector cue, next, direction cue, and finally, go cue) were presented. The $700 \mathrm{~ms}$ delay period between the "Direction cue" and "Go cue" was considered the phase of directional movement planning. EEG data segment within this period was used for further analysis.

Auditory feedback was given to help the subjects fulfill the instructions correctly. Four different tones were used to mean "correct", "error", "early", and "time out", 
respectively. In the reach tasks, if the point on the screen touched by the subject was outside the boundary of a $\left(5.5^{\circ} \times 5.5^{\circ}\right)$ square centered on the target cross, the "error" tone sounded. If the response began during the movement preparation period ( 0 $700 \mathrm{~ms}$ after direction cue onset), the "early" warning sounded. The "time out" feedback sounded when response time was $>500 \mathrm{~ms}$. All other trials were followed by the "correct" feedback sound. Only those trials are considered here. Subjects were instructed to perform tasks accurately to achieve a high score (percentage of correct trials). Their score was displayed on the screen at the end of each block. Some practice blocks were run before starting the EEG recording. For each subject, the experiment consisted of four blocks (with breaks in between) each including five runs of 45 trials. Within each block, there was a 20 -second rest between runs. A total of 900 trials were equally distributed between the nine tasks, which were presented to the subject in a pseudorandom sequence.

\subsection{Data Recording}

EEG data were recorded using $\mathrm{Ag} / \mathrm{AgCl}$ electrodes from 128 scalp positions distributed over the entire scalp using a BioSemi ActiveTwo EEG system (Biosemi, Inc.). Eye movements were monitored by additional bipolar horizontal and vertical EOG electrodes. All signals were amplified and digitized at a sample rate of $256 \mathrm{~Hz}$. Electrode locations were measured with a 3-D digitizer system (Polhemus, Inc.). Three cue presentation events and two manual response events ("button release" and "screen touch") were recorded on an event channel synchronized to the EEG data by DataRiver software (A. Vankov).

\subsection{Data Processing and Analysis}

Here, we only focused on estimations of planned direction of movement. Therefore we first separated the trials for each subject into three classes (left, right, and center) for offline analysis. In each class, the three tasks with different effectors (hand, eye, both) were mixed together. Investigation of effector-specific (hand or eye) EEG activations will not be included in this paper.

Data were analyzed using tools in the EEGLAB toolbox [11]. Epochs from the response delay period, 0 to $700 \mathrm{~ms}$ following direction cue onset, were extracted from the continuous data, and labeled by movement direction. The period $[0,100 \mathrm{~ms}]$ was used as baseline for each trial. Electrodes with poor skin contact were identified by their abnormal activity patterns and then removed from the data. For each subject, electrode locations were co-registered with a spherical four-shell head model used for dipole source localization.

\section{Spatial Filtering}

Independent component analysis (ICA) has been widely used in EEG analysis [1214]. It can decompose the overlapping source activities constituting the scalp EEG 
into functionally specific component processes. Here, we used ICA as an unsupervised spatial filtering technique to extract parietal EEG independent component (IC) activities that excluded noise from eye and muscle components as well as brain activities from other functional processes (e.g., in motor, visual, and frontal areas). For each subject, all trials were band-pass filtered (1-30 Hz), concatenated, and then decomposed using the extended infomax ICA algorithm [15]. Two lateralized temporo-parietal components were easily identified in each subject's decomposition by their spatial projections and significant contributions to the average event-related potential (ERP) waveforms time locked to onsets of the movement direction cue.

Figure 2 shows the scalp projections of the two parietal component clusters for all four subjects, plus their mean scalp maps. Clustering was done based on IC scalp maps using EEGLAB tools. These components contributed most to the scalp ERPs obtained by averaging the channel data over all the trials. To indicate the anatomical source location of these components, IC maps were subjected to equivalent dipole localization using the EEGLAB plug-in DIPFIT [11]. Source locations were specified in the Talairach coordinate system. Equivalent dipole localization (average Talairach coordinates: $[-33,-59,28]$ in the left hemisphere and $[40,-49,30]$ in the right hemisphere) indicated that these IC sources originated from the PPC (Brodmann Area 39/40). These results demonstrate that the PPC is activated during intended movement planning. To further explore the underlying neural mechanism of direction coding in the PPC, the parietal ICs were selected and back-projected onto the scalp to visualize their separate contributions to the scalp data.
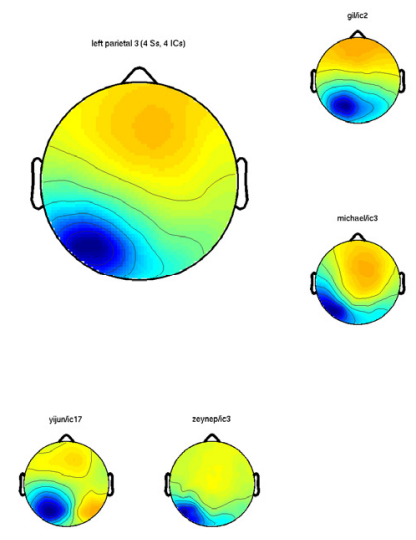

(a)
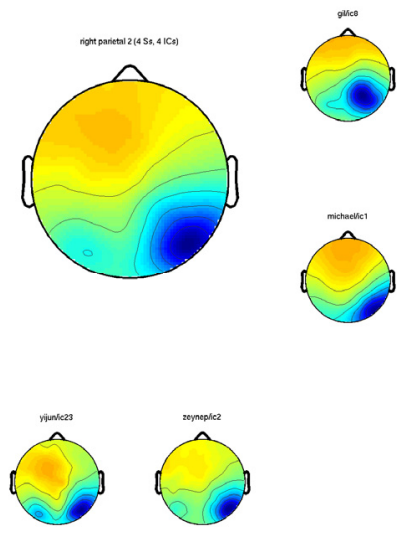

(b)

Fig. 2. Two clusters of lateralized temporo-parietal components with equivalent dipole locations in the (a) left hemisphere and (b) right hemisphere. Large cartoon heads show the mean scalp map for each cluster. Small heads show the clustered component maps for each of the four subjects. 


\section{ERP Modulation}

To extract the direction-specific portion of the ERPs, we compared the spatiotemporal patterns of the parietal EEG components for the different movement directions. For all four subjects, we found a consistent hemispheric asymmetry over the parietal cortex during the delay period $(0-700 \mathrm{~ms}, 0-100 \mathrm{~ms}$ used as baseline) in which motor planning can be presumed to have continued until cued movement onset (after 700ms). The projected PPC ICs produced a significant contralateral negativity and ipsilateral positivity with respect to intended movement direction. Scalp maps of left, right, and center classes for one subject were shown in Fig.3. For the "left" and "right" classes, their maps showed significant ipsilateral positivity. For instance, the left hemisphere has much higher amplitude than the right hemisphere when planning left movements. For the "center" condition, the map has a symmetric distribution on both sides and the amplitudes are much lower compared to "left" and "right" conditions. To further investigate the time course of this hemispheric asymmetry, difference wave was calculated by subtracting the contralateral activity from the ipsilateral activity with respect to movement direction. Two electrodes with highest weights in the two parietal IC maps were selected to represent the left and right hemispheres. In the difference wave averaged across the "left" and "right" trials, the hemispheric asymmetry was characterized by two contralateral negativities peaking $200 \mathrm{~ms}$ and $320 \mathrm{~ms}$ after the direction cue respectively, with mean amplitudes of $1.9 \mu \mathrm{V}$ and $3.8 \mu \mathrm{V}$ across subjects (see Fig.4).
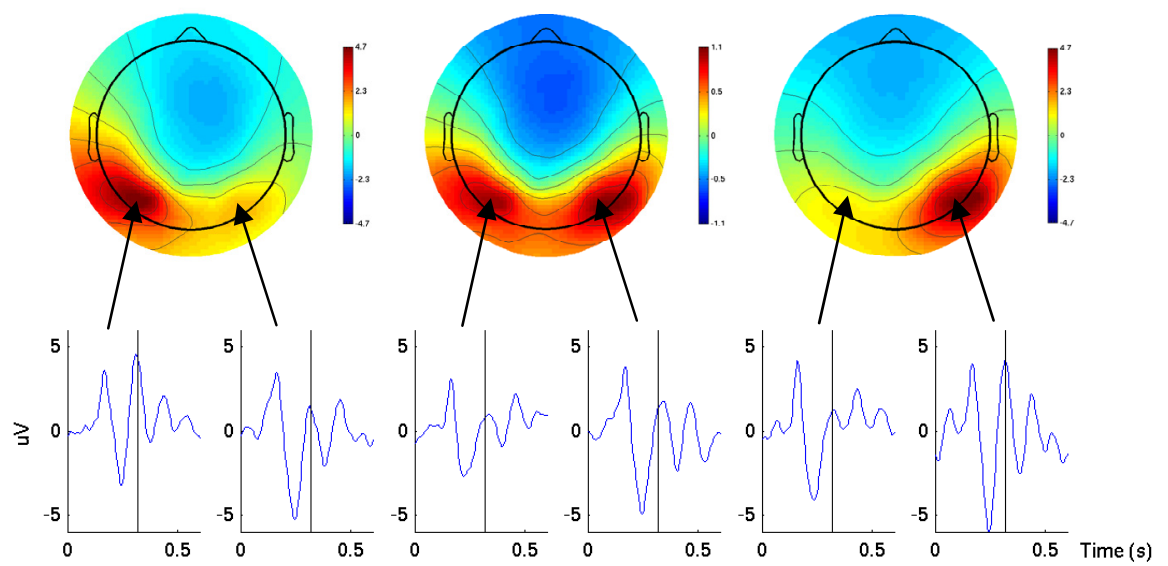

Fig. 3. Scalp maps and ERP waveforms of the summed, back-projected parietal ICs for one subject in the three different direction conditions (left, center, and right) at $320 \mathrm{~ms}$ after the direction cue. Note that the color scales of the scalp maps differ. The ERP waveforms were from two lateral parietal electrodes with strongest PPC projections. 


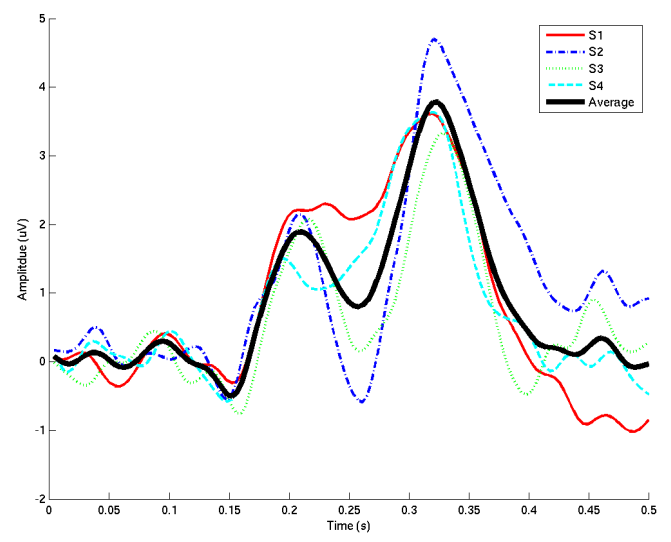

Fig. 4. Ipsilateral minus contralateral difference waves averaged over the "left" and "right" trials. Two peaks centered at $200 \mathrm{~ms}$ and $320 \mathrm{~ms}$ were the most significant hemispheric asymmetries appearing during planning of directional movements.

\section{Feature Extraction and Classification}

As a first evaluation of the potential use of EEG activity in PPC for driving a BCI system, binary classification of "left" versus "right" trials was performed using standard machine learning techniques that have been successfully employed in current BCI research [16-18]. Because this study focused on EEG modulation in the parietal cortex, only the parietal IC components were used for feature extraction, although other cortical ICs might contribute separate information for classification of intended direction (e.g., somatomotor components). Although subjects were instructed not to make any response during the movement planning period, covert eye and muscle movements might have occurred, giving additional EEG signals informative for classifying movement direction contained in ICs accounting for eye or scalp muscle activities. Here we constrained the classification performance to reflect only the directional EEG information generated in parietal cortex. Subject-specific time- and frequency-domain parameters were derived for classification. A sliding window was used to optimize the latency and frequency windows giving best classification performance. Because we found that the low-frequency activity contributed to the classification for all subjects, for simplicity a low-pass filter was used to extract the frequency components. The selected time/frequency parameters were listed in Table.1. Not unexpectedly, optimized time windows are consistent with the time course character of the difference wave shown in Fig.4.

After low-pass filtering, normalized amplitudes in the selected time window, normalized at each time point to have a range of [-1 1] across trials, were employed as features. Feature vectors from both parietal components were concatenated and then 
input to a support vector machine (SVM) classifier using an RBF kernel. The SVM algorithm was performed using the LIBSVM toolbox [19]. 10x10-fold cross validation was run to estimate classification performance.

\section{RESULTS}

We used classification accuracy to evaluate classification performance. An average accuracy of $80.25 \pm 2.22 \%$ was obtained for single-trial classification across the four subjects. The classification results are listed in Table 1. Considering that this paradigm is based on single-trial classification, the accuracy is comparable to most current BCI systems, e.g., the P300-based and motor imagery-based BCIs. Moreover, subject variety (reflected in the standard deviation across the four subjects of only $2.22 \%$ ) does not appear to be as large as in other BCI system reports, suggesting that this method might be usable for more subjects than the other BCI systems. Testing this impression would doubtless require more subjects. These results suggest that more refined measures of movement intention-related EEG activity arising in the PPC (and elsewhere in cortex) might be used to build a robust and noninvasive BCI system.

Table 1. Time-frequency parameters and classificatioin performance for all subjects.

\begin{tabular}{cccc}
\hline Subject & Time Window $(\mathrm{ms})$ & Frequency Window $(\mathrm{Hz})$ & Accuracy $(\%)$ \\
\hline S1 & $180-500$ & $0-25$ & $79.9 \pm 0.45$ \\
S2 & $150-480$ & $0-25$ & $81.9 \pm 0.94$ \\
S3 & $180-450$ & $0-20$ & $77.2 \pm 0.86$ \\
S4 & $210-510$ & $0-35$ & $81.9 \pm 0.81$ \\
\hline Mean & - & - & $80.25 \pm 2.22$ \\
\hline
\end{tabular}

\section{CONCLUSION AND DISCUSSIONS}

In this EEG study, we designed a movement delay paradigm to investigate brain activities in the human PPC during planning of intended movements. The results indicated that EEG signals generated in the PPC are altered during movement planning, and their hemispheric asymmetries carry information about intended movement direction. By analyzing multi-channel ERPs at the single-trial level, we obtained stable classification of "go left" and "go right" planning trials for all subjects. The resulting classification accuracy of $80.25 \%$ makes this paradigm promising for BCI design. 
Classification performance might be improved by considering the following factors. First, during motor planning, the PPC also encodes effector information, producing effector-specific brain activity patterns [20]. In the current data analysis, three tasks with different kinds of effectors (hand, eye, and both) were not distinguished, and may introduce variance linked to the different effectors used. Therefore, classifying trials involving the same effector might be more efficient. Else, a multi-factorial classification scheme might be used that included information as to the intended effector. Finally, the same data might be able to predict both the intended effector and movement direction. Second, for feature extraction a simple sliding window was used to select the latency window and frequency band used. To find more informative parameters, time-frequency decomposition methods might be applied allowing additional selection of optimal time-frequency measures. Third, additional features derived from EEG power modulation may be complementary to current features obtained from the time-domain waveforms. For example, [21] showed that the direction of visuospatial attention could be predicted by measuring alpha band power over the two posterior brain hemispheres.

Several potential applications of this paradigm may be expected. It could be directly used to implement a BCI based simply on decoding movement direction. Else, it could be integrated into current BCI systems to realize more robust or multidimensional control. For example, combining this paradigm with a motor imagerybased BCI (using EEG changes linked to imagining movements of left hand and right hand), might double the number of selective commands (from 2 to 4). Else, motor imagery of left and right hand movements might be linked to different directions (e.g., by imagining the left hand pointing to the left, or the right hand pointing to the right). In this case, by introducing additional parietal EEG components to mu/beta components from sensorimotor areas, classification performance can be significantly increased, although in this case the system would remain a two-class mode.

Before implementing a practical online BCI system based on intended movement direction, several issues still need further investigation. First, changes in attention and intention both contribute to direction-related EEG modulation. To learn more details about the relationship between these two factors, standard spatial attention experiments might be used to identify purely intention-related features. Else, some combination of subject attention and intention might give more efficient directionspecific brain patterns for a BCI communication or control system. In a practical system, movement planning without subsequent motor activity might be associated with lower BCI performance. The participants in this study were healthy volunteers; a direct test of the system concept on patients with motor disabilities will therefore be necessary before proposing applications for subjects with motor disabilities.

\section{References}

1. Lebedev, M.A., Nicolelis, M.A.L.: Brain-Machine Interfaces: Past, Present and Future. Trends in Neurosciences 29(9), 536--546 (2006) 
2. Taylor, D.M., Tillery, S.I.H., Schwartz, A.B.: Direct Cortical Control of 3D Neuroprosthetic Devices. Science 296, 1829--1832 (2002)

3. Nicolelis, M.A.L.: Actions from Thoughts. Nature 409, 403--40 (2001)

4. Hochberg, L.R., Serruya, M.D., Friehs, G.M., Mukand, J.A., Saleh, M., Caplan, A.H., Branner, A., Chen, D., Penn, R.D., Donoghue, J.P.: Neuronal ensemble control of prosthetic devices by a human with tetraplegia. Nature 442(7099), 164-171 (2006)

5. Quiroga, R.Q., Snyder, L.H., Bastista A.P., Andersen R.A.: Movement Intention Is Better Predicted than Attention in the Posterior Parietal Cortex. J. Neurosci. 26(13), 3615--3620 (2006)

6. Wolpaw, J.R., Birbaumer, N., Heetderks, W.J., McFarland, D.J., Peckham, P.H., Schalk, G., Donchin, E., Quatrano, L.A., Robinson, C.J., Vaughan, T.M.: Brain-Computer Interface Technology: A Review of the First International Meeting. IEEE Trans. Rehabil. Eng., 8, 164--173 (2000)

7. Wolpaw, J.R., Birbaumer, N., McFarland, D.J., Pfurtscheller, G., Vaughan, T.M.: BrainComputer Interfaces for Communication and Control. Clinical Neurophysiology 113(6), 767--791 (2002)

8. Birbaumer, N.: Breaking the Silence: Brain-Computer Interfaces (BCI) for Communication and Motor Control. Psychophysiology 43, 517--532 (2006)

9. Hammon, P.S., Makeig, S., Poizner, H., Todorov, E., de Sa, V.R.: Predicting Reaching Targets from Human EEG. IEEE Signal Processing Magazine 25(1), 69--77 (2008)

10. Waldert, S., Preissl, H., Demandt, E., Braun, C. Birbaumer, N., Aertsen, A., Mehring, C.: Hand Movement Direction Decoded from MEG and EEG. J. Neurosci. 28(4), 1000--1008 (2008)

11. Delorme, A., Makeig, S.: EEGLAB: An Open Source Toolbox for Analysis of SingleTrial EEG Dynamics Including Independent Component Analysis. J. Neurosci. Meth. 134, 9--21 (2004)

12. Makeig, S., Westerfield, M., Jung, T.P., Townsend, J., Courchesne, E., Sejnowski, T.J.: Dynamic Brain Sources of Visual Evoked Responses. Science 295, 690--694 (2002)

13. Jung, T.P., Makeig, S., McKeown, M.J., Bell, A.J., Lee, T.W., Sejnowski, T.J.: Imaging Brain Dynamics Using Independent Component Analysis. Proc. IEEE. 89, 1107--1122 (2001)

14. James, C.J., Hesse, C.W.: Independent Component Analysis for Biomedical Signals. Physiol. Meas. 26, R15--39 (2005)

15. Lee, T.W., Girolami, M., Sejnowski, T.J.: Independent Component Analysis Using an Extended Infomax Algorithm for Mixed Subgaussian and Supergaussian Sources. Neural Comput. 11(2), 417--441 (1999)

16. Lotte, F., Congedo, M., Lecuyer, A., Lamarche, F., Arnaldi, B.: A Review of Classification Algorithms for EEG-Based Brain-Computer Interfaces. J. Neural Eng. 4, R1--13 (2007)

17. Müller, K.R., Krauledat, M., Dornhege, G., Curio, G., Blankertz, B.: Machine Learning Techniques for Brain-Computer Interfaces. Biomed. Tech. 49(1), 11--22 (2004)

18. Kaper, M., Meinicke, P., Grossekathoefer, U., Lingner, T., Ritter, H.: BCI Competition 2003-Data Set IIb: Support Vector Machines for the P300 Speller Paradigm. IEEE Trans. Biomed. Eng. 51(6), 1073--1076 (2004)

19. Chang, C., Lin, C.: LIBSVM : a Library for Support Vector Machines. Software available at http://www.csie.ntu.edu.tw/ cjlin/libsvm (2001)

20. Calton, J.L., Dickinson, A.R., Snyder, L.H.: Non-Spatial, Motor-Specific Activation in Posterior Parietal Cortex. Nat. Neurosci. 5, 580--588 (2002)

21. Thut, G., Nietzel, A., Brandt, S.A., Pascual-Leone, A.: Alpha-Band Electroencephalographic Activity over Occipital Cortex Indexes Visuospatial Attention Bias and Predicts Visual Target Detection. J. Neurosci. 26(37), 9494--9502 (2006) 